\title{
Goos-Hänchen shift of Cosine- Gaussian Schell-model beams with rectangular symmetry
}

M. A. Berbel, A. Cunillera, R. Martínez-Herrero 


\title{
Goos-Hänchen shift of Cosine-Gaussian Schell-model beams with rectangular symmetry
}

\author{
M.A. Berbel ${ }^{a}$, A.Cunillera ${ }^{b}$, R. Martínez-Herrero ${ }^{a}$ \\ ${ }^{a}$ Departamento de Óptica, Universidad Complutense de Madrid, Ciudad Universitaria, 28040 \\ Madrid, Spain; \\ ${ }^{b}$ Departamento de Matemática Aplicada, Universidad Complutense de Madrid, Ciudad \\ Universitaria, 28040 Madrid, Spain
}

\begin{abstract}
In this contribution we study the relation between the second order intensity moments and the Goos-Hänchen shift for partially coherent totally polarized beams. The results are applied to a type of partially coherent beams, the Cosine-Gaussian Schell-model beams with rectangular symmetry.
\end{abstract}

Keywords: Goos-Hänchen shift, second order intensity moments, partially coherent beams

\section{INTRODUCTION}

A beam reflected off an interface experiences spatial and angular shifts from the geometrical-optics picture which depend on the polarization and the beam profile. Displacements in the plane of incidence are known as the Goos-Hänchen (GH) shift whereas the Imbert-Fedorov (IF) shift occurs in the plane orthogonal to the plane of incidence. Both shifts have been extensively studied theoretical and experimentally for a wide number of beam configurations and interfaces. ${ }^{1-20}$ In the present work we obtain closed and simple expressions that provide the relation between the GH shift and the global spatial structure of a totally polarized partially coherent beam, within the framework of the second-order irradiance moments. ${ }^{21-26}$ This paper is organized as follows. In section 2 , the formalism and the key definitions of second order intensity moments are introduced. The relation between the GH shift and the second order intensity moments is investigated in section 3 . Finally, in section 4 , we analyze the GH shift for a Cosine-Gaussian Schell-model beam with rectangular symmetry.

\section{INTENSITY SECOND ORDER MOMENTS FORMALISM}

It has long been established that the second-order coherence properties of a beam can be described by means of the cross-spectral density (CSDF) function $W\left(\boldsymbol{r}_{1}, \boldsymbol{r}_{2}, z\right)$, where $\boldsymbol{r}_{j}, j=1,2$ represent the two-dimensional position vectors at two points over the beam cross-section, transverse to the propagation direction $z$. Since we will consider quasi-monochromatic fields, explicit dependence on frequency $\omega$ will be omitted in our expressions for simplicity. Instead of analysing the structure of the light field by means of the function $W$ here we are interested on the global behaviour of the beam described by certain overall parameters that propagate according to simple laws. Let us then introduce the Wigner distribution function (WDF) associated with the CSDF function through a Fourier transform relationship:

$$
h(\boldsymbol{r}, \boldsymbol{\eta}, z)=\int W\left(\boldsymbol{r}+\frac{\boldsymbol{s}}{2}, \boldsymbol{r}-\frac{\boldsymbol{s}}{2}\right) \exp (i k \boldsymbol{\eta} \cdot \boldsymbol{s}) d \boldsymbol{s}
$$

where the dot symbolizes the inner product and $k \boldsymbol{\eta}=(k u, k v)$ provides the wavevector components along the $\mathrm{x}$ and $\mathrm{y}$-axes (accordingly, $u$ and $v$ represent angles of propagation, without taking the evanescent waves into account). In terms of the WDF, the so-called beam irradiance moments (denoted by sharp brackets) can be defined as follows ${ }^{22}$

Further author information: (Send correspondence to R.Martínez-Herrero) R.Martínez-Herrero: E-mail: r.m-h@fis.ucm.es

Third International Conference on Applications of Optics and Photonics, edited by Manuel F. M. Costa, Proc. of SPIE Vol. 10453, 104530C · C 2017 SPIE · CCC code: 0277-786X/17/\$18 · doi: 10.1117/12.2271487 


$$
\left\langle x^{m} y^{n} u^{p} v^{q}\right\rangle(z)=\frac{1}{I} \int x^{m} y^{n} u^{p} v^{q} h(\boldsymbol{r}, \boldsymbol{\eta}, z) d \boldsymbol{s} d \boldsymbol{r}
$$

where $m, n, p$ and $q$ are integer numbers and $I$ is proportional to the total beam power. The four first-order moments, $\langle x\rangle,\langle y\rangle,\langle u\rangle$ and $\langle v\rangle$, characterize the centre of the beam and its mean direction while the resulting ten different second-order moments allow a global and meaningful characterization of the spatial structure of a partially coherent quasimonochromatic beam. For instance, $\langle x y\rangle(\langle u v\rangle)$ gives the orientation of the principal axis (absolute axis) through the condition $\langle x y\rangle=0(\langle u v\rangle=0)^{27-29}$ and the crossed moments $\langle x v\rangle$ and $\langle y v\rangle$ carry out information about the twist and the orbital angular momentum of the beam. ${ }^{22,28,29}$ Taking into account that in paraxial approach the Wigner function satisfies $h(\boldsymbol{r}, \boldsymbol{\eta}, z)=h(\boldsymbol{r}-z \boldsymbol{\eta}, \boldsymbol{\eta}, 0)$ the free propagation law of the second-order moments is very simple and enables us to obtain the information at any $z$ from the knowledge of the second-order moments at arbitrary but fixed initial plane namely $z=0$. At this point it is interesting to note that this parameters can be directly written in terms of the CSDF, for instance we have

$$
\begin{gathered}
\left\langle u^{2}\right\rangle=\frac{\iint\left(\frac{\partial^{2} W\left(\boldsymbol{r}_{1}, \boldsymbol{r}_{2}\right)}{\partial x_{1} \partial x_{2}}\right)_{r_{1}=\boldsymbol{r}_{2}=\boldsymbol{r}} d x d y}{k^{2} \iint W(\boldsymbol{r}, \boldsymbol{r}) d x d y} \\
\langle x u\rangle=\frac{\iint x\left(\frac{\partial W\left(\boldsymbol{r}_{1}, \boldsymbol{r}_{2}\right)}{\partial x_{2}}-\frac{\partial W\left(\boldsymbol{r}_{1}, \boldsymbol{r}_{2}\right)}{\partial x_{1}}\right)_{r_{1}=\boldsymbol{r}_{2}=\boldsymbol{r}} d x d y}{2 i k \iint W(\boldsymbol{r}, \boldsymbol{r}) d x d y}
\end{gathered}
$$

The advantage of the above expressions is that they enable us to obtain the second order intensity moments from the knowledge of the CSDF without using any modal beam expansion. ${ }^{30-33}$

\section{GOOS-HÄNCHEN SHIFT MODEL}

We examine optical reflection at a planar interface, $z=0$ separating two media. Let us consider a quasimonochromatic totally polarized coherent beam, the electric field of such beam referred to a Cartesian frame attached to the incident beam $\left(x_{I}, y_{I}, z_{I}\right)$ can be written as

$$
\boldsymbol{E}\left(\boldsymbol{r}_{I}, z_{I}\right)=\boldsymbol{E}_{0} A\left(\boldsymbol{r}_{I}, z_{I}\right)
$$

Where $\boldsymbol{E}_{0}=\left(a_{1}, a_{2}\right)$ is a complex vector carrying the information of the state of polarization and $A\left(\boldsymbol{r}_{I}, z_{I}\right)$ is the amplitude of the beam at point $\boldsymbol{r}_{I}$ belonging to a transverse plane perpendicular to $z_{I}$ axis. It has been demonstrated ${ }^{13}$ that when a totally polarized coherent field is reflected off the surface each vector field $\boldsymbol{E}_{I}\left(\boldsymbol{r}_{I}, z_{I}\right)$ (referred to the Cartesian frame $\left(x_{R}, y_{R}, z_{R}\right)$ attached to the reflected beam) transforms into

$$
\boldsymbol{E}_{R}\left(\boldsymbol{r}_{R}, z_{R}\right)=\left(a_{1} r_{1} A\left(-x_{R}+x_{1}, y_{R}-y_{1}, z_{R}\right), a_{2} r_{2} A\left(-x_{R}+x_{2}, y_{R}-y_{2}, z_{R}\right)\right)
$$

In the above equation $r_{j}, j=1,2$, are the Fresnel reflection coefficients evaluated at the incident mean angle $\theta_{0}$ for parallel and perpendicular polarization, while $x_{j}, y_{j}$ are the four possibly-complex shifts defined as

$$
x_{j}=-\frac{i}{k}\left(\frac{\partial \ln r_{j}}{\partial \theta}\right)_{\theta=\theta_{0}}, j=1,2
$$




$$
\begin{gathered}
y_{1}=\frac{i a_{2}}{a_{1} k}\left(1+\frac{r_{2}}{r_{1}}\right) \cot \theta_{0} \\
y_{2}=\frac{-i a_{1}}{a_{2} k}\left(1+\frac{r_{1}}{r_{2}}\right) \cot \theta_{0}
\end{gathered}
$$

Accordingly, the intensity distribution of the reflected beam becomes

$$
I_{R}\left(\boldsymbol{r}_{R}, z_{R}\right)=\sum_{j=1}^{2}\left|a_{j} r_{j}\right|^{2}\left|A\left(-x_{R}+x_{j}, y_{R}-y_{j}, z_{R}\right)\right|^{2}
$$

Then, the spatial $\Delta$ and angular $\Theta$ GH shifts are defined ${ }^{13}$ in terms of the first order moment of the reflected intensity in the following way

$$
\begin{gathered}
\Delta_{G H}=\left\langle x_{R}\right\rangle(0) \\
\Theta_{G H}=\frac{\partial\left\langle x_{R}\right\rangle(z)}{\partial z}
\end{gathered}
$$

Let us now consider a partially coherent totally polarized uniformly beam propagating along the axis $z_{I}$, such a beam is represented by his $2 \times 2$ cross-spectral density matrix (CSDM), $\hat{W}_{I}\left(\boldsymbol{r}_{1 I}, \boldsymbol{r}_{2 I}, z_{I}\right)$, of the form

$$
\hat{W}_{I}\left(\boldsymbol{r}_{1 I}, \boldsymbol{r}_{2 I}, z_{I}\right)=\boldsymbol{E}_{0}^{\dagger} \boldsymbol{E}_{0} W\left(\boldsymbol{r}_{1 I}, \boldsymbol{r}_{2 I}, z_{I}\right)
$$

Where $\boldsymbol{E}_{0}=\left(a_{1}, a_{2}\right)$ contains the information of the state of polarization as before and the cross-spectral density function (CSDF) of the beam $W\left(\boldsymbol{r}_{1 I}, \boldsymbol{r}_{2 I}, z_{I}\right)$, determines the coherence between two points $\left(\boldsymbol{r}_{j I}, j=1,2\right)$ belonging to a transverse plane perpendicular to $z_{I}$ axis.

When we deal with partially coherent beams, it has been proved ${ }^{31-33}$ that a genuine, i.e., non-negative definite cross-spectral density function can be expressed in the form

$$
W\left(\boldsymbol{r}_{1 I}, \boldsymbol{r}_{2 I}, z_{I}\right)=\int L^{*}\left(\boldsymbol{r}_{1 I}, \boldsymbol{\sigma}, z_{I}\right) L\left(\boldsymbol{r}_{2 I}, \boldsymbol{\sigma}, z_{I}\right) d \boldsymbol{\sigma}
$$

where function $L(\boldsymbol{r}, \boldsymbol{\sigma}, z)$ satisfiesthe paraxial wave equation for each $\boldsymbol{\sigma}$. Eq. (11) has an interesting physical interpretation: Since the integrand has a factorized structure, it can be thought as the CSDF of a completely coherent field and consequently the overall CSDF is the continuous superposition of uncorrelated coherent contributions. By using Eq. (11) The CDSM of incident field becomes

$$
\hat{W}_{I}\left(\boldsymbol{r}_{1 I}, \boldsymbol{r}_{2 I}, z_{I}\right)=\int \mathbf{U}^{\dagger}\left(\boldsymbol{r}_{1 I}, \boldsymbol{\sigma}, z_{I}\right) \mathbf{U}\left(\boldsymbol{r}_{2 I}, \boldsymbol{\sigma}, z_{I}\right) d \boldsymbol{\sigma}
$$

Where the row vector $\mathbf{U}$ is given by

$$
\mathbf{U}\left(\boldsymbol{r}_{I}, \boldsymbol{\sigma}, z_{I}\right)=\left(a_{1}, a_{2}\right) L\left(\boldsymbol{r}_{I}, \boldsymbol{\sigma}, z_{I}\right)
$$

Given a fixed $\boldsymbol{\sigma}$, Eq. (12) is analogous to Eq. (4) and the formalism developed for totally coherent beams can be applied. Therefore, when the field is reflected off the surface, each vector field $\mathbf{U}\left(\boldsymbol{r}_{I}, \boldsymbol{\sigma}, z_{I}\right)$ transforms into 


$$
\mathbf{U}\left(\boldsymbol{r}_{R}, \boldsymbol{\sigma}, z_{R}\right)=\left(a_{1} r_{1} L\left(-x_{R}+x_{1}, y_{R}-y_{1}, \boldsymbol{\sigma}, z_{R}\right), a_{2} r_{2} A\left(-x_{R}+x_{2}, y_{R}-y_{2}, \boldsymbol{\sigma}, z_{R}\right)\right)
$$

Accordingly, the intensity distribution of the reflected beam becomes

$$
I_{R}\left(\boldsymbol{r}_{R}\right)=\sum_{j=1}^{2}\left|a_{j} r_{j}\right|^{2} \int\left|L\left(-x_{R}+x_{j}, y_{R}-y_{j}, \boldsymbol{\sigma}, z_{R}\right)\right|^{2} d \boldsymbol{\sigma}
$$

Starting from Eq. (14), after having performed the integration in the transverse spatial coordinates and taken into account Eq. (11) and the definition of second order intensity moments we obtain the following expression for the GH shift ${ }^{34}$

$$
\begin{gathered}
\Delta_{G H}=\sum_{j=1}^{2} w_{j}\left(\operatorname{Re}\left(x_{j}\right)+2 k\langle x u\rangle(0) \operatorname{Im}\left(x_{j}\right)+2 k\langle x v\rangle(0) \operatorname{Im}\left(y_{j}\right)\right) \\
\Theta_{G H}=2 k \sum_{j=1}^{2} w_{j}\left(\left\langle u^{2}\right\rangle \operatorname{Im}\left(x_{j}\right)+\langle u v\rangle \operatorname{Im}\left(y_{j}\right)\right)
\end{gathered}
$$

where $w_{j}$ is the fraction of the reflected intensity with polarization $j,^{35}$

$$
w_{j}=\frac{\left|a_{j} r_{j}\right|^{2}}{\sum_{j=1}^{2}\left|a_{j} r_{j}\right|^{2}}
$$

\section{GOOS-HÄNCHEN SHIFT OF COSINE-GAUSSIAN SCHELL-MODEL BEAMS WITH RECTANGULAR SYMMETRY}

In this section we consider a recently introduced new type of partially coherent beams, the Cosine-Gaussian Schell-model beams with rectangular symmetry ${ }^{36}(\mathrm{CGSM})$, the CSDF of such beam reads

$$
W\left(x_{1}, y_{1}, x_{2}, y_{2}\right)=F_{x}\left(x_{1}, x_{2}\right) F_{y}\left(y_{1}, y_{2}\right)
$$

The explicit expression of $F_{x}$ and $F_{y}$ being

$$
\begin{aligned}
& F_{x}\left(x_{1}, x_{2}\right)=\exp \left(-\frac{x_{1}^{2}+x_{2}^{2}}{4 \sigma_{0}^{2}}\right) \exp \left(-\frac{\left(x_{2}-x_{1}\right)^{2}}{2 \delta_{x}^{2}}\right) \cos \left(\frac{\sqrt{2 \pi} m\left(x_{2}-x_{1}\right)}{\delta_{x}}\right) \\
& F_{y}\left(y_{1}, y_{2}\right)=\exp \left(-\frac{y_{1}^{2}+y_{2}^{2}}{4 \sigma_{0}^{2}}\right) \exp \left(-\frac{\left(y_{2}-y_{1}\right)^{2}}{2 \delta_{y}^{2}}\right) \cos \left(\frac{\sqrt{2 \pi} m\left(y_{2}-y_{1}\right)}{\delta_{y}}\right)
\end{aligned}
$$

Here $\sigma_{0}^{2}$ denotes the r.m.s. transverse beam width, $\delta_{x}, \delta_{y}$ denote the transverse coherence widths along the $x$ and $y$ directions and $m$ is the beam order parameter. When $m=0$ this source reduces to the conventional Gaussian-Schell model source ${ }^{37,38}$

For this kind of beam it can be proven that the second order intensity parameters reads

$$
\langle x u\rangle=\langle x v\rangle=\langle y u\rangle=\langle y v\rangle=\langle u v\rangle=0
$$

and 


$$
\left\langle u^{2}\right\rangle=\frac{1}{4 k^{2} \sigma_{0}^{2}}\left(1+4 \sigma_{0}^{2}\left(\frac{1+2 \pi m^{2}}{\delta_{x}^{2}}\right)\right)
$$

Therefore by applying the model developed in section 3, we obtain the following expressions for the GH shifts

$$
\begin{gathered}
\Delta_{G H}=\sum_{j=1}^{2} w_{j} \operatorname{Re}\left(x_{j}\right) \\
\Theta_{G H}=2 k\left\langle u^{2}\right\rangle \sum_{j=1}^{2} w_{j} \operatorname{Im}\left(x_{j}\right)
\end{gathered}
$$

From the above expressions along with Eq. (20) we conclude that for this kind of beams and for a given interface the spatial GH shift only depends on the mean angle $\theta_{0}$ and the polarization characteristics of the beam. On the other hand, the angular GH shift depends on the polarization characteristics, the mean angle $\theta_{0}$ the transverse coherence width along the $\mathrm{x}$ direction and the beam order parameter. In figure 1 the behaviour of $\Theta_{G H}$ is plotted as a function of the degree of coherence $\beta=\delta_{x} / \sigma_{0}$ assuming an incidence angle greater than the Brewster angle. For both paralell and perpendicular polarization, the angular GH shift is greater in the case of a coherent incident beam $(\beta=0.1)$ than for low coherence $(\beta=10)$ or incoherent beams $(\beta=100)$ which was to be expected ${ }^{14}$. Furthermore, this model predicts increasing shifts for higher values of the order parameter $m$ if the incident beam is coherent and equal non-zero shifts if the incident beam is incoherent. The dependence of $\Theta_{G H}$ on the incidence angle when $\beta$ is fixed is illustrated in figure 2. It is observed that the order of the beam $m$ only involves a proportionality factor.

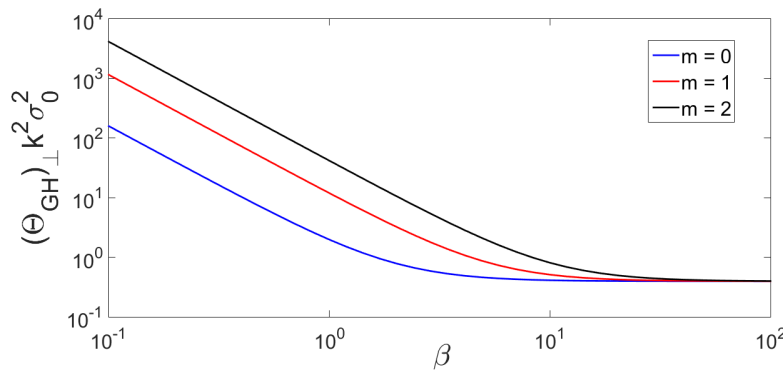

(a) Perpendicular polarization

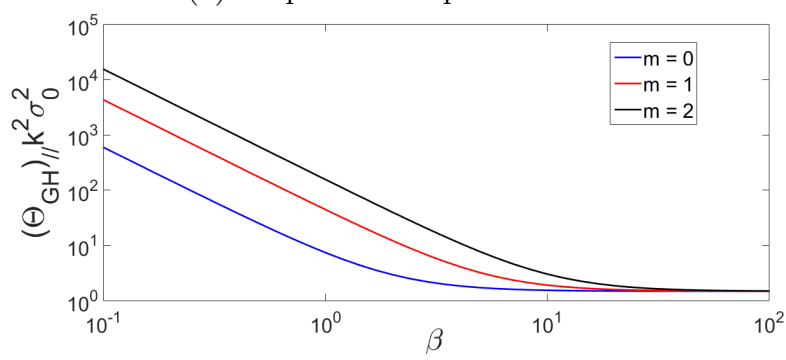

(b) Parallel polarization

Figure 1: Angular GH shift of CGSM beams with rectangular symmetry $(m=0,1,2)$ entering a planar interface with incidence angle greater than the Brewster angle. Refraction indices $n_{1}=1, n_{2}=1.514$. Incidence angle: $\theta_{0}=70^{\circ}$.

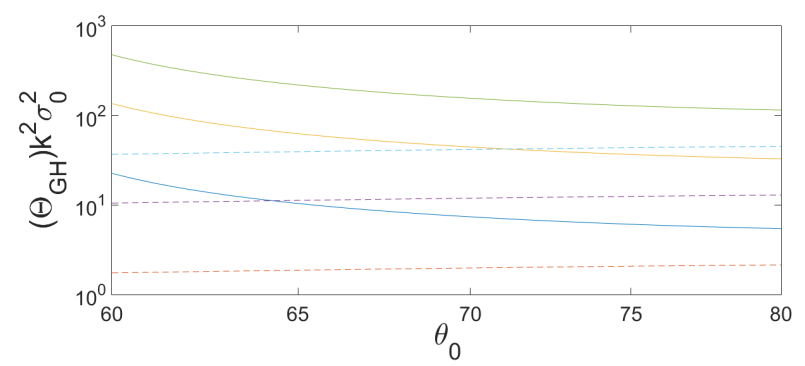

Figure 2: Angular GH shift dependence of CGSM beams with rectangular symmetry ( $m=0,1,2)$ with the incidence angle $\theta_{0}$. Parallel polarization (perpendicular is dotted), fixed coherence $\beta=1$, refraction indices $n_{1}=1, n_{2}=1.514$. Higher values of $m$ involve greater angular shifts. 


\section{ACKNOWLEDGMENTS}

Spanish Ministerio de Economía y Competitividad Project FIS2016-75147

\section{REFERENCES}

[1] Goos, F. and Hänchen, H., "Ein neuer und fundamentaler versuch zur totalreflexion," Annalen der Physik 436(7-8), 333-346 (1947).

[2] Artmann, K., "Calculation of the lateral shift of totally reflected beams," Ann. Phys. 437, 87-102 (1948).

[3] Fedorov, F. I., "K teorii polnogo otrazheniya," DOKLADY AKADEMII NAUK SSSR 105(3), 465-468 (1955).

[4] Imbert, C., "Calculation and experimental proof of the transverse shift induced by total internal reflection of a circularly polarized light beam," Physical Review D 5(4), 787 (1972).

[5] Bliokh, K. Y. and Bliokh, Y. P., "Polarization, transverse shifts, and angular momentum conservation laws in partial reflection and refraction of an electromagnetic wave packet," Physical Review E 75(6), 066609 (2007).

[6] Bliokh, K. Y. and Bliokh, Y. P., "Conservation of angular momentum, transverse shift, and spin hall effect in reflection and refraction of an electromagnetic wave packet," Physical review letters 96(7), 073903 (2006).

[7] Hosten, O. and Kwiat, P., "Observation of the spin hall effect of light via weak measurements," Science 319(5864), 787-790 (2008).

[8] Aiello, A. and Woerdman, J., "Role of beam propagation in goos-hänchen and imbert-fedorov shifts," Optics letters 33(13), 1437-1439 (2008).

[9] Li, C.-F., "Unified theory for goos-haenchen and imbert-fedorov effects," Physical Review A 76(1), 013811 (2007).

[10] Merano, M., Aiello, A., Van Exter, M., and Woerdman, J., "Observing angular deviations in the specular reflection of a light beam," Nature Photonics 3(6), 337-340 (2009).

[11] Merano, M., Hermosa, N., Woerdman, J., and Aiello, A., "How orbital angular momentum affects beam shifts in optical reflection," Physical Review A 82(2), 023817 (2010).

[12] Aiello, A. and Woerdman, J., "Role of spatial coherence in goos-hänchen and imbert-fedorov shifts," Optics letters 36(16), 3151-3153 (2011).

[13] Aiello, A., "Goos-hänchen and imbert-fedorov shifts: a novel perspective," New Journal of Physics 14(1), 013058 (2012).

[14] Löffler, W., Aiello, A., and Woerdman, J., "Spatial coherence and optical beam shifts," Physical review letters 109(21), 213901 (2012).

[15] Wang, L.-G., Zhu, S.-Y., and Zubairy, M. S., "Goos-hänchen shifts of partially coherent light fields," Physical review letters 111(22), 223901 (2013).

[16] Araújo, M. P., De Leo, S., and Maia, G. G., "Closed-form expression for the goos-hänchen lateral displacement," Physical Review A 93(2), 023801 (2016).

[17] Santana, O. J., Carvalho, S. A., De Leo, S., and de Araujo, L. E., "Weak measurement of the composite goos-hänchen shift in the critical region," Optics Letters 41(16), 3884-3887 (2016).

[18] Prajapati, C. and Ranganathan, D., "Goos-hanchen and imbert-fedorov shifts for hermite-gauss beams," JOSA A 29(7), 1377-1382 (2012).

[19] Ziauddin, Y.-L. C., Qamar, S., and Lee, R.-K., "Goos-hänchen shift of partially coherent light fields in epsilon-near-zero metamaterials," Scientific reports 6 (2016).

[20] Yin, X., Hesselink, L., Liu, Z., Fang, N., and Zhang, X., "Large positive and negative lateral optical beam displacements due to surface plasmon resonance," Applied Physics Letters 85(3), 372-374 (2004).

[21] Lavi, S., Prochaska, R., and Keren, E., "Generalized beam parameters and transformation laws for partially coherent light," Applied optics 27(17), 3696-3703 (1988).

[22] Serna, J., Martínez-Herrero, R., and Mejías, P., "Parametric characterization of general partially coherent beams propagating through abcd optical systems," JOSA A 8(7), 1094-1098 (1991).

[23] Martinez-Herrero, R. and Mejías, P., "Beam characterization through active media," Optics communications 85(2-3), 162-166 (1991). 
[24] Martínez-Herrero, R., Mejias, P., and Weber, H., "On the different definitions of laser beam moments," Optical and quantum electronics 25(6), 423-428 (1993).

[25] Mejías, P. and Martínez-Herrero, R., "Time-resolved spatial parametric characterization of pulsed light beams," Optics letters 20(7), 660-662 (1995).

[26] ISO 11146:2005 parts 1, . and 3, "Lasers and laser-related equipment-test methods for laser beam widths, divergence angles and beam propagation ratios," ISO Standard (2005).

[27] Serna, J., Mejías, P., and Martínez-Herrero, R., "Rotation of partially coherent beams propagating through free space," Optical and quantum electronics 24(9), S873-S880 (1992).

[28] Martínez-Herrero, R. and Mejías, P., "On the spatial orientation of the transverse irradiance profile of partially coherent beams," Optics express 14(8), 3294-3303 (2006).

[29] Martínez-Herrero, R. and Mejías, P., "On the control of the spatial orientation of the transverse profile of a light beam," Optics express 14(3), 1086-1093 (2006).

[30] Martínez-Herrero, R., "Expansion of complex degree of coherence," Il Nuovo Cimento B (1971-1996) 54(1), 205-210 (1979).

[31] Martínez-Herrero, R. and Mejías, P., "Radiometric definitions for partially coherent sources," JOSA A 1(5), $556-558$ (1984).

[32] Gori, F. and Santarsiero, M., "Devising genuine spatial correlation functions," Optics letters 32(24), 35313533 (2007).

[33] Martínez-Herrero, R., Mejías, P., and Gori, F., "Genuine cross-spectral densities and pseudo-modal expansions," Optics letters 34(9), 1399-1401 (2009).

[34] Martínez-Herrero, R., Berbel, M., and Cunillera, A. To be published .

[35] Martínez-Herrero, R., Mejías, P., Piquero, G., and Ramírez-Sánchez, V., "Global parameters for characterizing the radial and azimuthal polarization content of totally polarized beams," Optics Communications 281(8), 1976-1980 (2008).

[36] Liang, C., Wang, F., Liu, X., Cai, Y., and Korotkova, O., "Experimental generation of cosine-gaussiancorrelated schell-model beams with rectangular symmetry," Optics letters 39(4), 769-772 (2014).

[37] Mandel, L. and Wolf, E., [Optical coherence and quantum optics], Cambridge university press (1995).

[38] Serna, J., Mejias, P., and Martinez-Herrero, R., "Beam quality dependence on the coherence length of gaussian schell-model fields propagating through abcd optical systems," Journal of Modern Optics 39(3), 625-635 (1992). 